\title{
POLÍTICAS DE CIENCIA Y TECNOLOGÍA PARA LA PRODUCCIÓN INDUSTRIAL: MODELOS DE DESARROLLO Y SISTEMAS DE INNOVACIÓN EN ARGENTINA
}

\author{
IgNACIO DE ANGELIS \\ Universidad Nacional del Centro de la Provincia de Buenos Aires, \\ Argentina \\ deangelisignacio@gmail.com
}

\section{RESUMEN}

El concepto de capacidades tecnológicas para la producción permite evaluar las políticas públicas en ciencia y tecnología exponiendo la relación entre los sistemas de innovación y la estructura productiva de un país A partir de la identificación de tres etapas en la historia argentina reciente, el artículo propone un repaso sucinto por los distintos estadios en la configuración de esta relación durante los modelos de desarrollo adoptados desde 1930, prestando particular atención a la formación de capacidades científicas y tecnológicas en la industria manufacturera. Basado en una revisión conceptual y la propuesta analítica particular para el caso argentino, el artículo sostiene que la relación entre el sistema de innovación y los modelos de desarrollo devino en la formación de ciclos de fortalecimiento y divergencia, que limitaron el impulso y la consolidación de un Sistema Nacional de Innovación (SNI) dinámico e integrado a las fuerzas productivas industriales.

Palabras clave: Capacidades tecnológicas, Sistema nacional de innovación, Modelos de desarrollo, Argentina. 


\title{
SCIENCE AND TECHNOLOGY POLICIES FOR INDUSTRIAL PRODUCTION: DEVELOPMENT MODELS AND INNOVATION SYSTEMS IN ARGENTINA
}

\begin{abstract}
The concept of technological capacities for production permits the evaluation of public policies in science and technology by expounding on the relation between the innovation systems and the productive structure of a country. From the identification of three phases in recent Argentinian history, this article posits a succinct review of the different stages in the configuration of this relation during the development models adopted since 1930, paying particular attention to the formation of scientific and technological capacities in the manufacturing industry. Based on a conceptual review and the analytical proposal particular to the Argentinian case, the article asserts that a relation between the innovation system and the development models led to the formation of cycles of strengthening and divergence, which limited the impetus and the consolidation of a dynamic National System of Innovation (NSI) integrated into the industrial productive forces.
\end{abstract}

Keywords: Technological capacities, National system of innovation, Development models, Argentina. 


\section{INTRODUCCIÓN}

Desde fines de la década de 1980, los enfoques sistémicos para abordar el cambio tecnológico adquirieron centralidad en la instrumentación de políticas de innovación en los países centrales. Posteriormente, este marco analítico fue incorporado a los programas científicos y tecnológicos de los países periféricos en general, y en América Latina en particular. La adopción de estos enfoques expuso la necesidad de construir un esquema de interpretación que permita la instrumentación del marco para la acción de las políticas de innovación de acuerdo a la realidad se los países menos industrializados. En particular, y desde la perspectiva que asume este trabajo, el análisis de los sistemas de innovación debe interpretarse como una dimensión de la política industrial en su capacidad de reconvertir los esfuerzos en ciencia y tecnología para la producción de bienes y servicios y su inserción en el sistema internacional.

El artículo comienza con una síntesis conceptual del enfoque de sistemas de innovación como marco para el análisis y la formulación de las políticas en ciencia y tecnología, para luego avanzar en la propuesta para abordar el estudio del caso argentino sobre un conjunto de consideraciones particulares. Metodológicamente, se plantea el análisis desde dos dimensiones principales para agrupar el conjunto de variables de estudio: una primera dimensión para la caracterización y evaluación del complejo institucional científico y tecnológico, su interacción y su funcionalidad en términos productivos; y una segunda dimensión de contexto, delimitada por las características socioeconómicas que se desprenden del modelo de desarrollo adoptado en un momento histórico determinado. Para ello, se adopta la definición de modelos de desarrollo en términos regulacionistas (Boyer 2007, Neffa 2006, De Angelis, Calvento y Roark 2013), definido como una determinada configuración de las formas de regulación institucional del orden socioeconómico y su articulación con el régimen de acumulación (como formas de producción y consumo).

En el plano metodológico adquiere relevancia la búsqueda por avanzar hacia un enfoque idiosincrático para la evaluación de las políticas y su impacto en los procesos estructurales de las estrategias de desarrollo. La profundización del estudio del caso nacional requiere un grado de especificidad no siempre presente en la literatura que discute teóricamente estos enfoques desde el pensamiento latinoamericano en ciencia y tecnología (Arocena y Sutz 2000, Hurtado 2014). En efecto, la generalización regional de la problemática puede distorsionar las diferencias nacionales perdiendo especificidad en el diagnóstico. En este sentido, el conjunto de países agrupados en los análisis regionales presentan numerosas y profundas 
diferencias geográficas, demográficas, culturales, económicas, sociales y políticas. Por supuesto que estas diferencias replican en su devenir científico y tecnológico, cuya evolución reciente muestra trayectorias divergentes (Lamarchand 2010).

El enfoque propuesto destaca la pertinencia del análisis centrado en las capacidades tecnológicas, interpretadas en un sentido amplio desde un marco definido en la interacción de los distintos actores del sistema de innovación y producción y las diferentes formas de regulación institucional, económicas y políticas que adquiere el modelo de desarrollo. En este sentido, resulta un enfoque que prioriza las condiciones de contexto del modelo de regulación. Es decir, asume una perspectiva macro que incorpora la discusión en torno a las políticas públicas de regulación por sobre los elementos propios del comportamiento microeconómico y la conducta innovativa de las empresas en la formación de capacidades tecnológicas.

A partir de allí, en la segunda parte del artículo se avanza en un repaso histórico por la génesis y el desarrollo del sistema de innovación argentino, identificando su origen durante el modelo de industrialización por sustitución de importaciones y un recorrido divergente durante el período de desarticulación productiva neoliberal, el cual culmina con la constitución del sistema de innovación en términos formales durante la década del noventa.

En tercer lugar, se caracteriza el inicio de una nueva etapa a partir de las transformaciones implementadas en el marco de reactivación productiva luego de la crisis de la convertibilidad y del restablecimiento de la política científica y tecnológica, sustentada en la emergencia del nuevo modelo de desarrollo. De este modo, se sostiene la hipótesis de que la relación entre el sistema de innovación y los modelos de desarrollo se constituyó a partir de la formación de ciclos de fortalecimiento y divergencia que impidieron la consolidación de un Sistema Nacional de Innovación (SNI) dinámico e integrado a las fuerzas productivas industriales.

El artículo finaliza con el repaso de las principales evidencias empíricas disponibles para la caracterización de las capacidades científicas, tecnológicas y productivas, durante los últimos años, identificando los principales desafíos que debe asumir el Estado frente a un nuevo período de transformación del modelo de desarrollo. 


\section{UNA INTRODUCCIÓN A LA CONCEPTUALIZACIÓN DE LOS SISTEMAS DE INNOVACIÓN}

El devenir del siglo XX y la reconfiguración de la sociedad industrial a partir del surgimiento del nuevo paradigma tecnoeconómico de la información y la comunicación propusieron una ruptura en la visión tradicional de la ciencia y su relación con la sociedad ${ }^{1}$, transformando el modo de analizar la generación y la movilidad del conocimiento. En este contexto, e impulsados por el agotamiento del modelo lineal que inspiró las políticas de innovación centradas en el empuje de la oferta (Rosegger 1996, Bush 1999), en la última década del siglo XX comienzan a plantearse nuevos modelos interactivos (Kline y Rosenberg 1986) para promover políticas tendientes a compatibilizar las necesidades sociales y de mercado con la oferta tecnológica.

La inconsistencia del enfoque lineal en sus limitaciones para explicar la realidad y las diferencias económicas y tecnológicas entre los países, obligó a los teóricos y a los hacedores de política a interpretar los nuevos enfoques, surgidos principalmente de las experiencias nacionales de los entonces países centrales - miembros de la Organización para la Cooperación y el Desarrollo Económico (OCDE)-, que comenzaron a ser incorporados, durante los noventa, como recomendaciones formales en los documentos de la organización (Chudnovsky 1999, OCDE 1996).

Hacia fines de la década de 1980, y en la búsqueda de respuestas superadoras, surgió el concepto de sistemas de innovación ${ }^{2}$. Adaptándose a un escenario mundial caracterizado por la emergencia de un nuevo paradigma tecnoeconómico (Dosi 2003), la noción sistémica se basa en la idea de que la competitividad internacional de un país está directamente relacionada a la capacidad de su estructura productiva de adaptarse al cambio tecnológico (Lundvall 2009). Ello supone la aceptación del

1 El pensamiento tradicional se constituyó a partir de la obra de Robert Merton (1977) y la interpretación de la ciencia como una institución ahistórica y descontextualizada.

2 Navarro (2001) y Hervás et al. (2012) señalan como primer antecedente del concepto SNI, al trabajo de Freeman, "The National System of Innovation in historical perspective", de 1987, donde se identifica en el estudio de casos nacionales una relación causal entre el éxito de las innovaciones y su impacto en la productividad a partir de los procesos interactivos de las firmas con otros actores. A su vez, Freeman hace referencia a un antecedente previo de la concepción sistémica en el trabajo del economista alemán Friedrich List sobre los "Sistemas Nacionales de Economía Política” a mediados del siglo XIX. 
enfoque de ventajas dinámicas expuesto tempranamente por Schumpeter (1943), el cual sostiene que las ganancias de competitividad dependen de la capacidad de una economía por incorporar innovaciones y tecnología en sus formas de producción, lo cual determinará las ganancias internas y su forma de inserción internacional.

Para la caracterización del sistema de innovación, distintos autores (Edquist 1997, Lundvalll y Johnson 1994) señalan en la identidad del concepto su naturaleza holística e interdisciplinaria, su perspectiva histórica y su carácter no lineal e interdependiente, destacando la importancia de las instituciones y la función interpretativa que ofrece el concepto como categoría teórica.

Esta aproximación analítica se inscribe dentro de las teorías evolucionistas que, frente a las teorías neoclásicas, rechazan la idea de la tecnología como componente exógeno de los sistemas productivos y destacan la necesidad de un agente coordinador del desarrollo tecnológico (López 2002). El concepto sistémico enfatiza el carácter acumulativo del conocimiento y la innovación, destacando la importancia que tienen para una economía y su trayectoria tecnológica la magnitud y orientación de los esfuerzos formales e informales que se llevan a cabo, en tanto a partir de ellos es posible estimular el ritmo y la dirección del cambio técnico (Lugones 2000).

En términos evolucionistas el Estado deberá coordinar iniciativas para orientar los esfuerzos en el aumento de la competitividad de la economía, fortaleciendo la estructura de producción a partir del aumento de la productividad. De este modo, la función del Estado será la de promover, impulsar y fortalecer un $\mathrm{SNI}^{3}$ a través de distintos mecanismos e iniciativas, buscando la coordinación de políticas públicas en ciencia y tecnología con las demás políticas de desarrollo económico y social.

En este orden de análisis, conceptualmente, el SNI es entendido como el espacio definido por la coevolución de cinco subdominios institucionales: 1) la generación de conocimiento científico; 2) el desarrollo, mejora y adaptación de tecnologías y técnicas de producción; 3) la estructura económica y las formas de producción y distribución de bienes y servicios; 4) la estructura legal y política; y 5) el dominio cultural, entendido como

3 Si bien la concepción de SNI remite necesariamente a los procesos interactivos que tienen lugar dentro de las fronteras de un determinado Estado nación (Lundvall 1992), aportes posteriores permiten sobre la misma base sistémica del enfoque diferenciar sistemas de innovación regionales o locales (Yoguel 2000), e incluso sectoriales (Malerba 2002). 
el conjunto normas, valores y costumbres que moldean una sociedad (Freeman 2008).

Es necesario señalar que, si bien se destaca el Estado como principal agente coordinador y planificador mediante las políticas públicas, un SNI no se puede forjar deliberadamente ya que existen elementos que evolucionan de manera espontánea. En este sentido, se destaca el rol central de la estructura productiva y el régimen de acumulación (como pauta de producción y consumo) en la conformación del sistema de innovación. En efecto, el sistema adquiere ciertas características en función de la especialización productiva, y, a su vez, -lo que es más importante desde la perspectiva que se propone en este artículo- la especialización productiva de un país puede transformarse a partir de la consolidación de un sistema de innovación dinámico en su adaptación a los ritmos del cambio que propone el paradigma tecnoeconómico (Pérez 2001).

De esta manera, el enfoque reconoce que hacia el logro de competitividad no solo serán exitosas aquellas economías con capacidad para producir y generar conocimiento, sino que también dependerá de las capacidades de difusión, asimilación y aprendizaje (Gutti 2008), donde participan e interactúan todas las unidades del sistema.

El enfoque sistémico además de identificar el modo de producción de conocimiento desde una matriz microeconómica (principalmente basada en la firma) intenta comprender y problematizar los mecanismos y las formas en que el conocimiento se distribuye en el conjunto de la economía y en la sociedad. El elemento sustancial de este enfoque es el rol de las instituciones, no solo como marco para la interacción de los agentes y como estructura de incentivos (López 2002) sino como expresión misma de la evolución social en la adaptación del paradigma tecnoeconómico y su regulación social; es decir, expone la importancia de las instituciones como marco regulatorio y como expresión social de la dinámica tecnológica sobre el desarrollo.

Como sostienen López (2002), Kababe (2011) y Lugones (2005), el concepto de SNI no se traduce en una teoría formal normativa que permita generar pautas de comportamiento como recetas para la construcción de un sistema de innovación exitoso, o el establecimiento de parámetros rigurosos para medir o comparar empíricamente los distintos sistemas nacionales. Sin embargo, el enfoque ofrece lineamientos analíticos ordenados para la problematización de la relación entre desarrollo social y económico desde una perspectiva científica y tecnológica, permitiendo establecer distintos niveles de análisis a partir de la definición de un objeto 
de estudio, identificando los actores e interacciones claves para evaluar el desempeño del sistema en un tiempo determinado (Kababe 2011).

Chudnovsky sostiene que el enfoque se constituyó en un valioso aporte "para abordar y pensar la problemática de la innovación en países desarrollados y en desarrollo" (1999: 158) en tanto es lo suficientemente flexible para adaptarlo a realidades históricas e institucionales diversas. En igual sentido, López (2002) argumenta que al centrarse en los procesos y la interacción, y no solo en las condiciones estructurales, permite establecer estudios sobre las dinámicas propias de los países con menor grado de desarrollo. A su vez, para Freeman (1995) es posible pensar la aplicabilidad del concepto a los países con distinto grado de desarrollo y trayectorias tecnológicas, por lo que se deduce que cada país puede presentar un sistema de innovación más o menos rudimentario o desarrollado, según cada trayectoria particular.

En este sentido, la propuesta de un modelo analítico centrado en las particularidades nacionales en diálogo crítico con los estudios regionales generalistas (Arocena y Sutz 1999) requiere avanzar en la identificación y caracterización de los distintos actores y sectores con particularidades en la dinámica de innovación y producción, referenciando su interacción con los modelos de desarrollo. En efecto, la profundización del estudio del caso nacional requiere un grado de especificidad no siempre presente en la literatura que discute teóricamente estos enfoques, principalmente desde el pensamiento latinoamericano en ciencia y tecnología. Pues la generalización regional de la problemática puede distorsionar las diferencias nacionales perdiendo especificidad en el diagnóstico. El conjunto de países agrupados en los análisis regionales presentan numerosas y profundas diferencias geográficas, demográficas, culturales, económicas, sociales y políticas. Por supuesto que estas diferencias replican en su devenir científico y tecnológico, cuya evolución reciente muestra trayectorias diferentes (Lamarchand 2010).

Con todo, en el próximo apartado se presentan algunas consideraciones analíticas para el estudio del sistema de innovación argentino y su relación con los distintos modelos de desarrollo adoptados desde el siglo XX, haciendo foco en las relaciones que se producen con la dinámica productiva industrial. La centralidad puesta en el concepto de capacidades, permite avanzar en la propuesta de una metodología analítica para la identificación de las etapas del desarrollo científico y tecnológico desde la confrontación entre las principales características del sistema de innovación y la especificidad que adquiere el modelo de desarrollo vigente en cada período analizado. 


\section{CAPACIDADES CIENTÍFICAS, TECNOLÓGICAS Y PRODUCTIVAS COMO ELEMENTOS SUSTANTIVOS LA EVALUACIÓN DEL SISTEMA DE INNOVACIÓN ARGENTINO}

La adopción del enfoque de sistemas de innovación ofrece un marco para evaluar el desempeńo innovativo desde una perspectiva amplia a partir de la valoración del esfuerzo tecnológico en la generación de capacidades científicas y técnicas (Kababe 2011). Las capacidades tecnológicas son definidas como aquellas que "involucran el conocimiento y las habilidades necesarias para adquirir, usar, adaptar, mejorar y crear tecnología" (Gutti 2008: 8). Las capacidades de absorción e innovación constituyen los dos componentes centrales de las capacidades tecnológicas, y se encuentran asociadas no solo a la inversión en investigación y desarrollo ( $\mathrm{I}+\mathrm{D})$ sino a un conjunto amplio de elementos económicos, sociales, políticos y organizacionales.

Las capacidades de absorción -centrales en estructuras productivas periféricas- son definidas como la capacidad de un país para absorber e incorporar tecnología generada externamente. Este conjunto de capacidades que determinan el grado potencial para usar, imitar o copiar tecnología pueden ser entendidas en función de factores que van desde el nivel de educación que tiene un país y de las capacidades técnicas e institucionales para su incorporación, hasta motivaciones de prestigio y estima social (Gutti 2008, De Angelis 2015).

Sin embargo, este tipo de capacidades resulta difícil de operacionalizar metodológicamente, por lo tanto, en forma frecuente se utilizan indicadores de educación general y el número de profesionales en ciencia e ingeniería para dimensionar el nivel de los distintos países, lo cual -es necesario aclarar- refleja una mirada sesgada sobre la eventual capacidad de un país para incorporar y adaptar tecnología, principalmente porque desde el enfoque sistémico se incorpora una concepción amplia de las capacidades que atiende al conjunto de actores y la sinergia sistémica que pueda generarse en los esfuerzos por absorber tecnología, y que no resultan sencillo de sintetizar en un conjunto de indicadores, en tanto se constituyen en función del contexto social y las formas de regulación institucional, económicas y políticas del modelo de desarrollo vigente.

Por su parte, las capacidades de innovación refieren a las posibilidades potenciales que posee un país de generar conocimiento y desarrollar (o mejorar) nuevos productos, procesos o servicios de forma endógena. La capacidad para generar tecnología de un país, o una firma, constituye una fuente principal de competitividad. Para ello, será fundamental el 
desarrollo de elementos institucionales, sociales y políticos y la generación de recursos complementarios ${ }^{4}$.

La propuesta de centralidad en el concepto de capacidades, permite avanzar en la caracterización de los sistemas de innovación teniendo en cuenta un conjunto de variables ordenadas en dos dimensiones principales, que buscan ilustrar la interacción del sistema científico y tecnológico con los determinantes del desarrollo económico argentino. En primer lugar, se encuentran aquellas que se corresponden directamente con el complejo científico y tecnológico, sus capacidades y su desempeño, conformando la dimensión científico-tecnológica. Luego, en una dimensión de contexto, se presentan las variables de carácter sistémicas que según la definición indicada más arriba, serán las que terminen de definir un sistema de innovación en la interacción del complejo científico y tecnológico con la estructura productiva y los demás actores.

Entre las primeras se encuentran la conformación y los grados de interacción que se produce entre sus elementos. Allí, será importante para la generación de sinergia las relaciones que emergen a partir de la trama institucional que lo compone y, principalmente, el nivel de esfuerzos desarrollados en el fortalecimiento de las capacidades, tanto en sus recursos humanos como materiales. Asimismo, los recursos no solo deben ser evaluados por su volumen, sino que es importante ofrecer una caracterización a partir de la orientación de los esfuerzos concretos que son desplegados desde el sistema. Por último, se consideran los indicadores de resultado de patentes e innovaciones, a partir de las actividades, programas e iniciativas concretas ejecutadas en el marco del sistema, prestando atención a los esfuerzos científicos y tecnológicos de las empresas y sectores económicos.

A partir de allí, y sujetos a la razón sistémica que propone el enfoque, será necesario considerar un conjunto de variables complementarias correspondientes a la dimensión contextual, que son determinadas en gran medida por la estrategia de desarrollo económica y social adoptada. Así, el análisis de la estructura productiva y la influencia macroeconómica sobre las trayectorias tecnológicas resulta fundamental para comprender las dinámicas sistémicas.

En el siguiente esquema se presentan los tres niveles en que se aborda

4 iguiendo a Teece (2003), los recursos complementarios constituyen los elementos necesarios para implementar o comercializar la innovación, desde la etapa de fabricación hasta la etapa de difusión y distribución. 
el estudio nacional. En el primer nivel se encuentran las dos dimensiones centrales propuestas para el análisis, que, a partir de las categorías expuestas en el segundo nivel (complejo científico y tecnológico y modelo de desarrollo), dan forma al espacio común dónde se consolidan las capacidades del sistema. Este espacio común es definido por el SNI y se encuentra relacionado a un conjunto de variables que se presentan en el tercer nivel analítico.

Figura 1: Dimensiones para la caracterización del Sistema de Innovación argentino

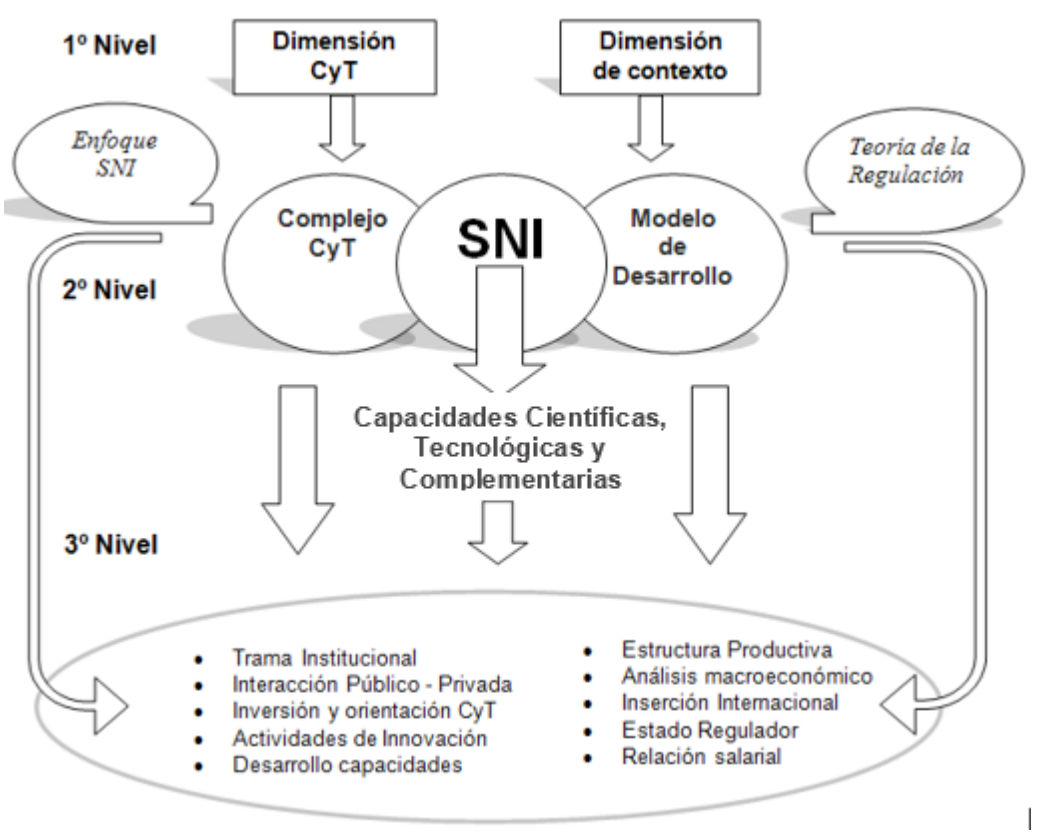

Fuente: Elaboración propia.

A partir de este esquema conceptual, a continuación se presenta una síntesis del recorrido histórico del sistema de innovación argentino y los distintos estadios del desarrollo, arribando, en la última sección, a un diagnóstico preliminar del período neodesarrollista sobre la base de un conjunto de datos empíricos correspondientes con el tercer nivel analítico, los cuales permiten sostener la argumentación en torno a la hipótesis propuesta de los ciclos de fortalecimiento y divergencia entre la dimensión científica y tecnológica y los modelos de desarrollo. 
ORIGEN Y DESARROLLO DEL SISTEMA DE INNOVACIÓN EN ARGENTINA: GÉNESIS EN LA ESTRATEGIA SUSTITUTIVA, FORMALIZACIÓN Y DIVERGENCIA NEOLIBERAL

Las instituciones inaugurales del actual complejo científico y tecnológico surgieron durante el modelo de desarrollo basado en la estrategia de industrialización por sustitución de importaciones (ISI), vigente en Argentina entre 1930 y 1976. Este modelo de desarrollo, en su perfil científico y tecnológico, se basó en la aplicación de incentivos para la generación de tecnología local y el control al ingreso de tecnología del exterior sobre dos áreas fundamentales: el sector productivo (recursos naturales, industrias y empresas estatales) y el sector militar (desarrollo nuclear, espacial y de armamentos).

Los primeros antecedentes de institucionalización estratégica a nivel nacional de la actividad científica y tecnológica se remontan a la década de 1940. En 1946 se creó la Secretaría Técnica de la Presidencia de la Nación, desde donde tuvo lugar la creación de la Comisión Nacional de Energía Atómica (CNEA) en 1950, y el Consejo Nacional de Investigaciones Técnicas y Científicas (CONITYC) en 1951 como la primera institución dedicada enteramente a actividades científicas y técnicas (CONICET 2006).

Luego, durante la década del cincuenta, se fue constituyendo el eje central del actual sistema de innovación con la creación del Instituto Nacional de Tecnología Agropecuaria (INTA), el Instituto Nacional de Tecnología Industrial (INTI), y la reconversión en 1958 del CONITYC en el Consejo Nacional de Investigaciones Científicas y Técnicas (CONICET). Asimismo, desde mediados de la década del cuarenta y hasta fines de los cincuenta fueron creadas otras instituciones y organismos, como el Instituto Antártico Argentino (IAA), el Instituto de Investigaciones Científicas y Técnicas de las Fuerzas Armadas (CITEFA), y en 1960 la Comisión Nacional de Investigaciones Espaciales, como así también tuvieron lugar desarrollos institucionales a nivel provincial, como la Comisión de Investigaciones Científicas (CIC) en la Provincia de Buenos Aires.

Como señala Bisang (1995), estas instituciones fueron surgiendo a partir de iniciativas de la comunidad de investigadores y de funcionarios públicos, pero también como respuestas a problemas concretos del sector productivo frente al modelo sustitutivo, replicando formas organizativas de países industrializados, difundidas por organismos internacionales como la Organización de Estados Americanos (OEA), la Comisión Económica Para América Latina (CEPAL) y la Organización de las Naciones Unidas 
para la Educación, la Ciencia y la Cultura (UNESCO).

En este marco, la pauta signada por los desafíos tecnológicos del sistema productivo local se expresó en la importancia que evidenciaron en la tarea de $\mathrm{I}+\mathrm{D}$ y en el fortalecimiento e integración de proveedores locales, las grandes empresas estatales como Obras Sanitarias de la Nación, Ferrocarriles Argentinos y Yacimientos Petrolíferos Fiscales (YPF). Del mismo modo, en este campo y en el relacionamiento del poder militar en la esfera productiva tuvo un rol fundamental el Ministerio de Defensa.

En cuanto al desarrollo tecnológico a nivel privado, el sistema productivo, amparado en un esquema de protección pero de difícil acceso a la tecnología extranjera, se centró en cambios menores. Es a partir de entonces, que en un contexto de fuerte dependencia de componentes básicos de producción para sostener el modelo de desarrollo basado en la estrategia ISI, se fue generando una brecha en las capacidades competitivas internacionales que sostuvo la viabilidad de las empresas locales solo a partir del sistema de protección y orientación hacia el mercado interno.

Con todo, las instituciones científicas y tecnológicas creadas durante el modelo sustitutivo no respondieron a una planificación integral centralizada de largo plazo, sino que surgieron como respuesta a problemas puntuales o sectoriales del sistema productivo (INTI e INTA), a intereses de la comunidad de investigadores (CONICET) y del Ministerio de Defensa (CNEA y CITEFA). Asimismo, durante diferentes períodos y de acuerdo a diversas coyunturas políticas y económicas, estas instituciones se mantuvieron más cerca o más lejos de sus objetivos originales, actuando muchas veces de forma descoordinada y tomando distancia del sistema productivo (Bisang 1995).

El modelo sustitutivo comenzó a desarticularse en un contexto de limitaciones internas propias a la estrategia ISI y de grandes transformaciones a nivel global. Desde la década del setenta se fueron generando importantes cambios asociados al surgimiento del nuevo paradigma tecnoeconómico ${ }^{5}$

5 El concepto de paradigma tecnoeconómico es propuesto por Carlota Pérez para explicar la determinación de las revoluciones científicas y tecnológicas en las grandes oleadas de desarrollo de la economía internacional. En este marco, destaca cinco etapas históricas desde la primera Revolución Industrial hasta la actualidad, donde cada una se corresponde con un paradigma: la primera entre 1770-1880 y 1830-1840, caracterizada como de mecanización temprana; la segunda entre 1830-1840 y 18801890, marcada por el desarrollo del vapor y ferrocarriles; la tercera entre 1880-1890 y 1930-1940 ,a partir del desarrollo y difusión del acero, electricidad e ingeniería 
con el impulso de la electrónica y las nuevas tecnologías de la información y la comunicación, dando lugar a una revolución científico-tecnológica de carácter global. En relación directa con el nuevo paradigma, se produjo el cambio en los modos de organización de las empresas y la redefinición de los espacios y las pautas de producción y consumo, arrojando un nuevo escenario de competitividad internacional basado en el conocimiento, dando origen a un nuevo régimen de acumulación capitalista posfordista sostenido en un esquema de producción flexible y deslocalizado geográficamente (Freeman y Pérez 2003).

A nivel interno, a partir de 1976 la dictadura militar abandonó la estrategia de desarrollo basada en la ISI dando paso a un modelo de desarrollo neoliberal, que continuó, con matices, en los posteriores gobiernos democráticos y alcanzó su máxima expresión en la década del noventa durante la vigencia de la ley de convertibilidad cambiaria. Se produjo una importante transformación de la estructura económica nacional, desplazando a la industria como eje dinámico del sistema productivo y redirigiendo capitales hacia el mercado financiero.

Este proceso consolidó una estructura productiva especializada en actividades recursos-naturales-intensivas que benefició a los grandes grupos económicos nacionales y a las empresas transnacionales (Basualdo 2013). Las nuevas alianzas entre los grupos económicos locales y el capital extranjero derivaron en la concentración y extranjerización de la estructura productiva y del comercio exterior. De esta manera se forjó un nuevo perfil de inserción internacional vulnerable y dependiente (Araya y Colombo 2009).

En lo que respecta al campo científico y tecnológico, en un contexto de vulnerabilidad y crisis económicas recurrentes, la actividad tuvo un lugar marginal. El gobierno militar dio impulso a la investigación en temas nucleares y espaciales, pero lo hizo en un contexto de represión, intervención, desarticulación y aislamiento de las instituciones científicas, tecnológicas y de las universidades (Albornoz y Gordon 2011). Por su parte, el gobierno de Alfonsín (1983-1989) recibió como herencia un país en crisis, endeudado, con una profunda crisis fiscal y caída de la inversión, situación que se agudizó con la hiperinflación y licuó los recursos de las universidades y las principales instituciones, agudizando su aislamiento (Nun 1995). Durante el gobierno de Carlos S. Menem, el desarrollo

pesada; la cuarta etapa entre 1930 y 1970-1990, centrada en el petróleo y automóvil; y la etapa actual, 1970-1990 hasta la actualidad, caracterizada por las tecnologías información y comunicación (Pérez 2004). 
científico y tecnológico siguió teniendo un lugar marginal que coincidió, sin embargo, con un conjunto de iniciativas formales en torno al auge internacional de los modelos de planificación basados en la innovación.

A comienzos de la década del noventa los esfuerzos institucionales vuelven a aparecen en la agenda política con la promulgación de la Ley 23.877 de Promoción y Fomento de la Innovación Tecnológica en 1990, estableciendo un fondo de promoción y la creación de las Unidades de Vinculación Tecnológicas (UVT) con el objetivo de articulación público privada entre el complejo científico y tecnológico nacional y el sector productivo $^{6}$. Con estas iniciativas, aunque de alcance limitado por la agudización de las reformas regulatorias neoliberales, se inaugura un cambio de rumbo en los intentos de reconversión institucional, el cual se intensificó en la segunda mitad del decenio (Albornoz y Gordon 2011).

En 1996 se produjo el primer antecedente en la conformación formal del SNI con la creación del Gabinete Científico y Tecnológico (GACTEC) ${ }^{7}$, como marco interdisciplinario conformado por los distintos ministerios nacionales con el objetivo de consensuar políticas a largo plazo. Desde allí, en 1997 se lanzó el Plan Nacional Plurianual de Ciencia y Tecnología 19982000, donde por primera vez se propone el desarrollo y fortalecimiento del Sistema Nacional de Ciencia, Tecnología e Innovación (GACTEC 1997). Otra de las instituciones inaugurales es la Agencia Nacional de Promoción de la Ciencia y la Tecnología (ANPCYT) ${ }^{8}$, creada también en 1996

6 Otras iniciativas relevantes fueron el Programa de Modernización Tecnológica (1994) financiado por el Banco Interamericano de Desarrollo, la reestructuración del INTI, la creación del Sistema Nacional de Normas, Calidad y Certificación de la Secretaría de Industria (Chudnovsky y López 1996).

7 Desde la creación del Ministerio de Ciencia, Tecnología e Innovación Productiva (MINCYT) en 2007 el GATEC dejó de ser coordinado de la jefatura de ministros y pasó ser coordinado desde el MINCYT. Actualmente, y debido a su falta de funcionamiento, tiene un rol marginal en la estructura del SNI.

8 Actualmente dependiente del MINCYT, la ANPCYT es un organismo dedicado a la promoción del financiamiento de proyectos científicos, tecnológicos y de innovación productiva tendiente a mejorar las condiciones sociales, económicas y culturales en la Argentina. Su objetivo de creación fue el establecimiento y coordinación nacional de instrumentos de financiamiento y vinculación del sector privado a los centros de investigación. Actualmente se constituye a partir de cuatro fondos principales: Fondo para la Investigación Científica y Tecnológica (FONCYT), Fondo Tecnológico Argentino (FONTAR), Fondo Fiduciario de Promoción de la Industria del Software (FONSOFT) y Fondo Argentino Sectorial (FONARSEC) promueve 
desde la Secretaría de Ciencia y Tecnología, la cual se constituye como un importante organismo de financiamiento que se agrega a los fondos de las instituciones existentes.

Como señala Chudnovsky (1999), este impulso durante la segunda parte de la década del noventa por sistematizar formalmente las iniciativas y políticas científicas y tecnológicas a la luz de las experiencias de los países industrializados, contrasta con la política de laissez-faire en política económica que caracterizó al gobierno de Menem. Lo que se produjo fue un quiebre en los fundamentos formales de la estrategia para el desarrollo tecnológico, ya que, en realidad, la política de laissez-faire responde a la concepción ortodoxa de la tecnología como factor exógeno -centrada en la oferta-, que se incorporada al sistema productivo a partir de la apertura de la economía y el impulso a la Inversión Extranjera Directa (IED).

El argumento a favor de la modernización a partir de la apertura económica y la liberalización del régimen de IED se basó en que estas impulsarían una mayor competencia en el mercado local facilitando el acceso a la importación de tecnología moderna y capitales para competir internacionalmente9. Las IED y la desregulación sobre las empresas transnacionales a su vez promoverían la incorporación de conocimientos técnicos y organizacionales avanzados que potenciarían la modernización (Chudnovsky y López 1996).

Por lo tanto, la política económica de liberalización, desregulación y descentralización que caracterizó esta estrategia de desarrollo fue coherente con la debilidad deliberada en política científica y tecnológica del modelo neoliberal vigente desde 1976. Sin embargo, a partir de la intensificación de los citados esfuerzos durante la década del noventa, y el giro hacia una visión estratégica del SNI desde 1996, se produjo un quiebre en esta correspondencia que nos remite a las categorías analíticas propuestas por Amílcar Herrera (1971) sobre la relación divergente entre las políticas explícitas y las políticas implícitas para el desarrollo de la ciencia y la tecnología. La divergencia es clara: las políticas explícitas se manifestaron a comienzos de la década del noventa y específicamente a partir de 1996 en la orientación estratégica desde el Estado hacia el desarrollo y

el financiamiento de proyectos tendientes a mejorar las condiciones sociales y económicas en la Argentina.

9 La política de liberalización tuvo en este sentido un efecto moderado ya que si bien se produjo un importante ingreso de IED, estuvo orientado fundamentalmente hacia bienes no transables. 
fortalecimiento del SNI; por su parte, las políticas implícitas se sostuvieron en el dejar ser de la apertura económica y la modernización en base a la liberalización y desregulación del mercado de bienes, servicios y factores, que es coincidente con la visión tradicional ortodoxa y dependiente del desarrollo científico y tecnológico.

El auge de la innovación impulsada por la adopción del concepto de SNI en las políticas de planificación en un contexto de reducción del gasto público, privatización de las grandes empresas estatales, descentralización de responsabilidades, liberalización y flexibilización de la estructura productiva, resultó, sin embargo, en una reducción de la importancia relativa de la $\mathrm{I}+\mathrm{D}$ y las actividades científicas y tecnológicas (Pupato, Chudnovsky y López 2004). A su vez, el desplazamiento de la función estratégica del Estado, y el posicionamiento del mercado como asignador de recursos, derivaron en la profundización de la dependencia tecnológica producto de la incorporación de tecnología extranjera.

En este orden de análisis, Nochteff (2002) sostiene que la virtual ausencia presupuestaria de la política científica y tecnológica en Argentina durante el período neoliberal responde principalmente -aunque no de forma determinante ni excluyente- a la insuficiente demanda de innovaciones de los principales agentes económicos con mayor capacidad de influir en las decisiones políticas. Estos agentes obtuvieron beneficios extraordinarios a partir de un conjunto de "ventajas monopólicas no basadas en la innovación" (Nochteff 2002: 558), y por lo tanto, no necesitaron del desarrollo de un sistema de innovación, ni de la formación de recursos humanos calificados para la obtención de ventajas competitivas dinámicas, sino, por el contrario, que basaron su obtención de beneficios sobre la disponibilidad de recursos naturales y en ventajas artificiales otorgadas a partir de subsidios, esquemas preferenciales, protecciones, concentración de mercados y libertades para fijar precios.

De este modo se forjó un escenario de tensión en el intento por impulsar la consolidación del SNI bajo una lógica neoliberal y sobre lo base de instituciones creadas a partir del modelo sustitutivo. En este sentido, las instituciones fundamentales heredadas del complejo científico y tecnológico fueron pensadas, originadas y desplegadas en base al modelo de desarrollo centrado en la estrategia de industrialización y protección, y por lo tanto, su sobrevivencia al cambio de modelo expresó la agudización de su falta de articulación y vinculación con el sistema productivo, exponiendo los límites de la adaptación pretendida. 
A pesar de estos esfuerzos, las principales características que adquirió el sistema científico tecnológico durante la década de 1990 -todas ellas marcadas por la débil vinculación entre economía, sociedad, ciencia y tecnología- fueron: la desarticulación de las políticas de ciencia y tecnología respecto del sistema productivo; la superposición de funciones de los distintos organismos ${ }^{10}$; la baja participación del sector privado en las actividades de investigación y desarrollo; escasos niveles de inversión; desvinculación en la formación de recursos humanos respecto al sector productivo; déficit en los recursos orientados a las actividades científicotecnológicas; escasa participación de sectores basados en conocimiento en la estructura productiva; y deficiencias en la distribución nacional en términos de localización de los recursos científicos y tecnológicos (Lugones 2005, López 2005, Chudnovsky 1999, Katz 2000).

Desde la perspectiva del sector productivo manufacturero en relación con la tecnología, la política industrial y los instrumentos promotores de la innovación, estuvieron signados por un enfoque horizontal (Gordon 2011), con medidas que no discriminaron entre los distintos rubros, sino que estuvieron destinadas al desarrollo de una infraestructura general. Esto, implicó un significativo retroceso ante la planificación de las políticas industriales sectoriales (predominantes en la etapa ISI) que supieron impulsar la promoción de sectores considerados estratégicos.

Con todo, las reformas implementadas durante la década del noventa profundizaron el proceso de desindustrialización y el desplazamiento de los capitales hacia los servicios y las actividades intensivas en el uso de recursos naturales. En efecto, la industria pasó de representar el 25\% del PBI en la década de los setenta, a solo el 17\% hacia finales de los noventa (BCRA). Entre 1995 y 2001 el número de empresas en el sector manufacturero se redujo de 50.000 a 45.000, a razón de 2500 nuevas empresas por cada 3.500 que desaparecieron por año (Pupato, Chudnovsky y López 2004).

En este contexto, con resultados contradictorios a partir de la adopción y difusión formal de los nuevos enfoques propuestos por los organismos internacionales, se produce el quiebre con la visión ortodoxa de planificación, reconociendo la posibilidad y necesidad de orientar los esfuerzos a través de políticas públicas. Sin embargo, esta nueva concepción sistémica adquirió

10 Hacia mediados de los noventa existían un conjunto de por lo menos 400 instituciones públicas a nivel nacional, provincial y municipal relacionadas con las actividades de ciencia y tecnología; de ellas, cuatro instituciones heredadas del modelo sustitutivo concentraban cerca del ochenta por ciento de los recursos (CONICET 39\%, INTA 21\%, CNEA 11\% e INTI 6\%) (Bisang 1995). 
sustento en el siguiente período a partir de la crisis modelo de desarrollo neoliberal y la salida de la convertibilidad que posibilitaron la emergencia de un nuevo patrón de crecimiento y el establecimiento de un nuevo modelo de características neodesarrollistas ${ }^{11}$.

En la siguiente sección, se presenta un diagnóstico empírico analítico de situación del sistema de innovación tomando como punto de partida referencial el período precrisis neoliberal, para luego centrarse en la dinámica que adquirió con la recuperación económica posconvertibilidad en un contexto de transformación orientado a consolidar el SNI.

\section{DE LA DIVERGENCIA NEOLIBERAL A LA CONVERGENCIA NEODESARROLLISTA: FORTALEZAS, DEBILIDADES Y DESAFÍOS DEL SISTEMA DE INNOVACIÓN}

Con el objetivo de trazar un diagnóstico del período en primer lugar se exponen las principales iniciativas institucionales y las evidencias empíricas en torno al fortalecimiento de las capacidades científicas y tecnológicas. En segundo lugar, se exponen las capacidades que se derivaron del nuevo modelo de desarrollo en torno a la reactivación productiva industrial. Desde allí, en torno a la interacción entre las dos dimensiones analíticas, surgen los desafíos cardinales para el fortalecimiento del sistema

En el plano institucional, desde el año 2003 se impulsaron una serie de cambios en referencia al marco legislativo, organizacional y presupuestario del sistema científico y tecnológico nacional previsto en la Ley 25.467, sancionada en $2001^{12}$. Entre las iniciativas más importantes, y en línea con las trasformaciones del nuevo paradigma tecnoeconómico global

11 El neodesarrollismo refiere a las nuevas estrategias de acumulación centradas en el crecimiento del sector industrial con capacidad para competir internacionalmente en un contexto globalizado (diferencia sustancial con el desarrollismo, que impulsó la industrialización en un marco de protección). En una concepción amplia, reconoce la recuperación del Estado en su capacidad para intervenir en la dinámica de producción y acumulación (vía política fiscal y cambiaria) y redirigir la inversión y los beneficios desde los sectores más dinámicos hacia aquellos que necesitan incentivos para su funcionamiento, generando, a su vez, condiciones socioeconómicas equitativas (Porta y Bianco 2004, Curia 2011).

12 La Ley 25.467 establece el marco general del Sistema Nacional de Ciencia, Tecnología e Innovación; los objetivos de la política científica y tecnológica nacional; las responsabilidades del Estado Nacional; la estructura del Sistema; su planificación; el financiamiento de las actividades de investigación y desarrollo; la evaluación de las mismas; y las disposiciones especiales y generales. 
y la inserción internacional de las capacidades locales, se encuentran la sanción de Ley de Promoción de la Industria del Software, la creación de la Fundación Argentina de Nanotecnología y la Ley de Promoción de la Biotecnología Moderna. Posteriormente, durante el ańo 2007, tuvo lugar la creación del Ministerio de Ciencia, Tecnología e Innovación Productiva, posicionándose como el organismo rector en la nueva planificación sectorial con el objetivo de revertir la débil vinculación entre economía, sociedad, ciencia y tecnología.

Otras iniciativas tendientes a la consolidar el sistema de innovación fueron la sanción de la Ley de Financiamiento Educativo del año 2006; la transformación y expansión del sistema universitario a partir de la creación de catorce universidades de gestión pública entre 2002 y 2014; la puesta en marcha en el ańo 2009 del Fondo Argentino Sectorial en el marco de la política de fortalecimiento y vinculación con el sector productivo de la ANPCYT; la creación de la Secretaría de Articulación Científico Tecnológica del Ministerio de Ciencia, Tecnología e Innovación Productiva (MINCYT) y la de Planeamiento y Políticas en Ciencia, Tecnología e Innovación Productiva, con el objetivo de fomentar los vínculos entre los organismos académicos, las universidades e instituciones de $\mathrm{I}+\mathrm{D}$ con los actores del sistema productivo; y, hacía el final del período tuvo lugar la puesta en marcha del Plan Estratégico Argentina Innovadora 2020.

Por otro lado, existió un denso conjunto de instrumentos implementados y coordinados por los principales ministerios de la nación con el objetivo de generar y fortalecer las capacidades tecnológicas de la estructura productiva. En el relevamiento realizado para este artículo se analizaron los instrumentos asociados directamente con el sector industrial y aquellas actividades de innovación relacionadas con el sector manufacturero que se encuentran disponibles en la Base de Instrumentos para el Desarrollo Productivo (BIDP) elaborada por la Dirección de Coordinación de Políticas Productivas del Ministerio de Economía de la Nación.

Dentro de la BIDP se identificaron un total de 266 políticas de promoción productiva, donde es posible señalar más de 120 instrumentos que afectan directa o indirectamente la actividad industrial y las actividades de investigación y desarrollo. Estos programas constituyeron beneficios concretos a partir de aportes no reembolsables, beneficios fiscales e impositivos, asistencia técnica, capacitación y formación de recursos humanos, certificación de calidad (principalmente a través del INTI), financiación a través de distintas líneas de crédito, y promoción de exportaciones e importaciones. 
En cuanto a los esfuerzos materiales y los indicadores específicos, el gasto destinado a Actividades Científicas y Tecnológicas (ACT) y a I+D ${ }^{13}$ en relación al producto total de la economía argentina, a partir del 2005, bajo el nuevo régimen macroeconómico y la nueva estrategia de desarrollo, se evidenció el comienzo de un proceso de crecimiento de los indicadores, alcanzando un pico máximo durante el 2011.

Tabla 1: Evolución del gasto en ACT e I+D como porcentaje del PBI, e Inversión por investigador en miles de dólares, 1997-2013 ${ }^{14}$

\begin{tabular}{ccccccccccc} 
& 1997 & 1999 & 2001 & 2002 & 2003 & 2005 & 2007 & 2009 & 2011 & 2013 \\
\hline ACT & $0,50 \%$ & $0,52 \%$ & $0,48 \%$ & $0,44 \%$ & $0,46 \%$ & $0,52 \%$ & $0,60 \%$ & $0,66 \%$ & $0,73 \%$ & $0,66 \%$ \\
I+D & $0,41 \%$ & $0,45 \%$ & $0,42 \%$ & $0,38 \%$ & $0,41 \%$ & $0,46 \%$ & $0,50 \%$ & $0,59 \%$ & $0,64 \%$ & $0,62 \%$ \\
Inversión & & & & & & & & & & \\
$\begin{array}{c}\text { de US\$/ } \\
\text { Investigador }\end{array}$ & 33,03 & 32,32 & 28,20 & 9,69 & 12,18 & 17,20 & 22,57 & 30,10 & 38,33 & 46,42 \\
& & & & & & & & & &
\end{tabular}

Fuente: Red de Indicadores de Ciencia y Tecnología (RICYT).

Respecto a los recursos humanos dedicados a las ACT, Argentina presenta una sólida base que se expresa en un alto número de investigadores en relación al total de la Población Económicamente Activa (PEA), con valores superiores a la media regional. Hasta el año 2003, el número total de investigadores se mantuvo estable, y a partir allí se produce un crecimiento sostenido. Esta ampliación en torno al $80 \%$ en la base de recursos humanos, fue impulsada por un aumento del 140\% de los becarios y un $80 \%$ en la cantidad de investigadores. A su vez, como se señala en la Tabla 1, estuvo acompañada por el crecimiento sustancial de los esfuerzos materiales medido en la inversión en dólares por investigador.

Al considerar la orientación de los esfuerzos en cuanto a la capacidad de inserción de los investigadores en la estructura productiva (Tabla

13 Las ACT son definidas como aquellas directamente relacionadas con la generación, el perfeccionamiento, la difusión y la aplicación de conocimientos científicos y técnicos, incluye: $\mathrm{I}+\mathrm{D}$, difusión, formación de recursos humanos, servicios tecnológicos, entre otros. Por su parte $\mathrm{I}+\mathrm{D}$ refiere a cualquier esfuerzo sistemático por aumentar el conocimiento, y el uso de los mismos para nuevas aplicaciones, incluye: Investigación básica, aplicada y desarrollo experimental.

$14 \mathrm{Al}$ momento de elaboración de este artículo el último dato disponible corresponde al año 2013. 
2), se observó una caída en la vinculación en los sectores de empleo asociados a empresas, situación que explica la absorción gubernamental de investigadores producto de la revitalización de los organismos estatales del SNI. Esto representa un singular desafío en el avance del fortalecimiento sistémico. Durante los ańos de divergencia entre el sector productivo y el sistema de innovación, la caída en la participación en el sector productivo fue absorbida por el sector educativo. Esta dinámica fue revertida solo parcialmente en los últimos años, dinamizando el resto de instituciones públicas. En efecto, resulta importante señalar los bajos niveles de permeabilidad del sector productivo en la incorporación de recursos humanos ${ }^{15}$, y dado que los límites de incorporación en el sector público, se enciende una luz de alarma respecto de los investigadores formados y en formación.

Tabla 2: Investigadores por cada mil integrantes de la PEA, inversión por investigador en miles de dólares, y sector de empleo, 1997-2013

\begin{tabular}{|c|c|c|c|c|c|c|c|c|c|c|}
\hline & 1997 & 1999 & 2001 & 2002 & 2003 & 2005 & 2007 & 2009 & 2011 & 2013 \\
\hline Investigadores & 2,71 & 2,78 & 2,75 & 2,78 & 2,87 & 3,16 & 3,68 & 4,10 & 4,67 & 4,76 \\
\hline Gobierno & $24,8 \%$ & $25,5 \%$ & $24,8 \%$ & $25,5 \%$ & $25,0 \%$ & $28,6 \%$ & $29,9 \%$ & $31,0 \%$ & $29,6 \%$ & $29,7 \%$ \\
\hline Empresas & $12,8 \%$ & $10,8 \%$ & $9,4 \%$ & $8,9 \%$ & $8,8 \%$ & $9,6 \%$ & $8,6 \%$ & $7,5 \%$ & $6,8 \%$ & $5,07 \%$ \\
\hline $\begin{array}{l}\text { Educación } \\
\text { Superior }\end{array}$ & $61,3 \%$ & $62,4 \%$ & $63,9 \%$ & $63,7 \%$ & $64 \%$ & $59,6 \%$ & $59,5 \%$ & $59,6 \%$ & $62,2 \%$ & $64,0 \%$ \\
\hline $\begin{array}{l}\text { Organismos } \\
\text { privados sin } \\
\text { fines de lucro }\end{array}$ & $1,0 \%$ & $1,1 \%$ & $1,8 \%$ & $1,7 \%$ & $2,0 \%$ & $2,0 \%$ & $1,8 \%$ & $1,7 \%$ & $1,3 \%$ & $1,1 \%$ \\
\hline
\end{tabular}

Asimismo, la vinculación entre la universidad pública y el sector productivo no ha podido superar el sesgo academicista que presentan las primeras. En este sentido, a partir de los datos de la RICYT, es posible analizar la orientación en la formación de los investigadores y señalar la preeminencia de las Ciencias Naturales y Exactas durante todo el período (26,8\% hacia 2013), el crecimiento de las Ciencias Sociales (del 14,5\% en

15 En este contexto, desde 2012 el MINCYT implementó distintos programas financiando la incorporación de doctores por parte de empresas públicas y privadas. 
1997 a 22\% en 2013) y la baja participación que evidencian las áreas de mayor vinculación con el sector productivo nacional como las Ciencias Agrícolas (14,9\% a $10 \%$ en 2013$)$ y la Ingeniería y Tecnología $(20 \%$ a $17,7 \%$ entre 1997 y 2013).

Por su parte, la información empírica disponible sobre el gasto en I+D por objetivo socioeconómico en 2013 señala la caída en la participación del rubro producción y tecnología industrial a menos del 10\% del total, mientras que el destino producción y tecnología agrícola mantiene su participación en torno al $12 \%$ del total. Al observar la inversión en $\mathrm{I}+\mathrm{D}$ por disciplina científica en los últimos ańos, se ubican en primer lugar a las Ciencias Naturales y Exactas (21\% en 2013) y la Ingeniería y Tecnología (20,3\% en 2013), luego Ciencias Agrícolas, Ciencias y Ciencias Sociales (aproximadamente el 10\% cada una).

Respecto a los indicadores de resultado, es posible asumir dos espacios analíticos, por un lado, aquel que se desprende de los resultados de patentamiento derivado de la actividad innovadora. Por otro lado, en un nivel más amplio, es posible describir un cuadro de situación dinámico a partir del comportamiento de los distintos actores del sistema de innovación y producción a partir de los datos que se desprenden de las encuestas de innovación.

Respecto a las patentes, su vinculación e inserción sistémica internacional, se observa un comportamiento que aunque modesto, resulta positivo y estable Tabla 3).

Tabla 3: Indicadores de patentes en Argentina, 1997-2013

\begin{tabular}{cccccccccc} 
& 1997 & 1999 & 2002 & 2003 & 2005 & 2007 & 2009 & 2011 & 2013 \\
\hline $\begin{array}{c}\text { Número de patentes } \\
\text { otorgadas }\end{array}$ & 1.228 & 1.241 & 911 & 1.367 & 1.798 & 2.769 & 1.354 & 1.291 & 1.297 \\
$\begin{array}{c}\text { Tasa de dependencia } \\
\text { Tasa de }\end{array}$ & 6,11 & 6,18 & 5,77 & 4,75 & 3,99 & 5,12 & 6,77 & 6,00 & 6,42 \\
$\begin{array}{c}\text { autosuficiencia } \\
\begin{array}{c}\text { Coeficiente de } \\
\text { invención }\end{array}\end{array}$ & 0,14 & 0,13 & 0,14 & 0,17 & 0,20 & 0,16 & 0,12 & 0,14 & 0,13 \\
& 2,37 & 2,46 & 1,91 & 2,09 & 2,73 & 2,38 & 1,59 & 1,71 & 1,54
\end{tabular}

Fuente: RICYT. 
La tasa de dependencia expresa la relación entre patentes solicitadas por residentes y no residentes, los valores mayores a uno expresan que la mayoría son solicitadas desde el exterior, es decir que corresponde a invenciones desarrolladas fuera del país. Consecuentemente, el indicador de autosuficiencia, que mide la relación entre las patentes solicitadas por residentes y el total se mantiene en niveles bajos. Por último, el coeficiente de invención, que muestra el número de patentes solicitadas cada cien mil habitantes, se mantiene en valores relativamente bajos a partir del 2009.

En este orden de análisis se inscriben las consecuencias del ingreso desregulado de empresas trasnacionales producto de las reformas estructurales y la nueva dinámica de la economía mundial. Estas empresas presentan en general una estrategia de concentrar sus esfuerzos en I+D en sus casas matrices.

Por su parte, empresas locales se desarrollan en entornos donde el volumen del mercado financiero y de capitales implica límites de recursos para el desarrollo de actividades de innovación (López 2005). En los países centrales, las empresas consiguen financiamiento en los mercados de capitales; institución emergente que no presenta un gran desarrollo en la Argentina. Es decir, que los esfuerzos orientados desde las políticas en ciencia y tecnología deben ser complementados por razones organizacionales, financieras, macroeconómicas, de trama productiva e inserción internacional.

En este sentido, el patrón de especialización productiva concentrado en la producción y exportación de commodities industriales y agropecuarios que se profundizó durante los años noventa, agudizó la baja demanda y la débil vinculación del sector productivo respecto a la oferta de conocimiento. Respecto a este último punto, según los datos de la Encuesta Nacional sobre Innovación y Conducta Tecnológica (ENIT) del año 2005, las empresas industriales muestran una baja vinculación con instituciones del sistema de innovación, los cuales tienen lugar principalmente $-\mathrm{y}$ a partir de intercambios de información y cooperación-, con el INTI y las Universidades (INDEC 2008). Más allá de estos vínculos, la encuesta arrojó como resultado que el $90 \%$ de las empresas no tuvieron contacto con otros organismos o programas gubernamentales de ciencia y tecnología.

Por su parte, en los últimos años y bajo el contexto de reactivación productiva del nuevo patrón de crecimiento, existió un importante grado de avance en la materia. A partir de los datos de la Encuesta Nacional de Dinámica de Empleo e Innovación sobre el sector manufacturero para el período 2010-2012 (MINCYT 2015a), se evidencia que las empresas 
industriales muestran un mejor desempeño en actividades de innovación y una mayor vinculación con otros actores del sistema. Los datos señalan que del total de empresas industriales relevadas, el $54 \%$ se vinculó con al menos un agente del sistema. Este porcentaje se eleva si se consideran las empresas innovativas -es decir, aquellas que realizan esfuerzos en actividades de innovación-, de las cuales un $70 \%$ tienen vínculos con otros actores.

Al considerar los datos de la inversión destinada a actividades de innovación del sector industrial para los años 2010-2012 (MINCYT 2015a), el 62\% del total se explica por adquisición de maquinaria y equipos, cerca del $18 \%$ en $\mathrm{I}+\mathrm{D}$, y un $8 \%$ en diseño industrial e ingeniería interna, con una participación marginal de los destinos restantes. Estos valores evidencian una mejora respecto a los anteriores datos disponibles (Encuesta Nacional sobre Innovación y Conducta Tecnológica (ENIT) del INDEC), dónde para el año 2007 las empresas industriales distribuían sus principales esfuerzos en un $67,1 \%$ en maquinaria y equipo, un $14,3 \%$ en $\mathrm{I}+\mathrm{D}$ y $6,2 \%$ diseño industrial.

Sin embargo, este cuadro de situación expone un problema adicional, dado que la mayoría de las inversiones en máquinas y equipos son realizadas mediante compras externas. Durante el año 2011 la adquisición de máquinas y equipos de origen extranjero alcanzó un máximo del $64.78 \%{ }^{16}$ del total, lo cual evidencia un proceso estructural de formación exógena de capacidades tecnológicas.

Con todo, el diagnóstico del SNI durante el período señalado da cuenta de un importante capital científico y tecnológico pero con agudas limitaciones estructurales generadas en su génesis y en su desarrollo, principalmente en torno a la dinámica público-privada y la vinculación de la oferta pública de conocimiento con los agentes productivos.

En este sentido, el crecimiento de la estructura productiva industrial durante el período posconvertibilidad significó un elemento central en el aumento de capacidades para el desarrollo tecnológico. La ampliación del mercado y la extensión de la estructura industrial constituyeron elementos fundamentales para el impulso de capacidades complementarias. Sin embargo, para ponderar el efectivo desarrollo de las mismas y su asiento dentro de las formas de producción deben considerarse los esfuerzos en las actividades de innovación realizadas por las empresas.

16 Datos que se desprenden de observar la composición de la Inversión Bruta Interna Fija total provistos por el Centro de Estudios para la Producción del entonces Ministerio de Industria de la Nación. 
Según la encuesta sobre I+D en el sector empresario elaborada por el Ministerio de Ciencia y Tecnología (MINCYT 2015b), las empresas realizan un cuarto de la inversión en $\mathrm{I}+\mathrm{D}$ en términos nacionales $(\$ 5.000 .000 .000)$ y el $75 \%$ restante está ejecutado por instituciones de ciencia y tecnología, universidades públicas y privadas y organizaciones sin fines de lucro.

Indagando sobre estas evidencias al interior de la estructura productiva se encuentra que las grandes empresas son las que más aportan a la inversión total en I+D. En efecto, las firmas multinacionales realizaron inversiones en $\mathrm{I}+\mathrm{D}$ por un total de $\$ 2.300 .000 .000$ (47\% del esfuerzo total del sector empresario), por su parte las grandes empresas nacionales invirtieron $\$ 1.400 .000 .000$ (29\% del total). Sin embargo, al analizar el esfuerzo respecto al total de ingresos por ventas de las firmas (es decir, su conducta innovadora), se observa que las pequeńas y medianas empresas -con un total de \$330.000.000 - muestran un desempeño significativamente superior, invirtiendo más del $4 \%$ de sus ventas. Por su parte, las grandes empresas multinacionales y nacionales exponen un perfil rentista, reinvirtiendo en I+D solo el $0,8 \%$ y el $0,6 \%$ de sus ventas totales respectivamente.

Desde esta perspectiva, es importante destacar que entre los sectores industriales de mayor crecimiento durante el período prevalece un importante grado de extranjerización y concentración en torno a las firmas líderes, entre los más importantes se encuentran los sectores de alimentos, la metalurgia, la industria química, petrolera y la industria automotriz. Estas empresas integran cadenas globales de valor, articulando sus funciones de producción con proveedores extranjeros y concentrando sus principales inversiones en $\mathrm{I}+\mathrm{D}$ fuera del país. Asimismo, mediante la remisión de utilidades y las compras e insumos y bienes de producción en el extranjero, generan problemas en la disponibilidad de divisas de la economía argentina.

En este sentido, las firmas industriales que más recursos destinaron a $\mathrm{I}+\mathrm{D}$ consideradas de acuerdo a los rubros en los que se desempeñan, fueron las empresas del sector farmacéutico, seguidas por las semilleras, y sustancias y productos químicos. Estos tres sectores industriales intensivos en conocimiento, junto con el sector de servicios informáticos, representan más de la mitad del monto total destinado a $\mathrm{I}+\mathrm{D}$ por el sector productivo (MINCYT 2015b).

Con todo, se debe destacar que el avance durante los últimos años en los instrumentos e iniciativas públicas para la formación de capacidades científicas y tecnológicas fue acompañado por cambios de menor significancia en la conducta innovativa de las empresas. Asimismo, más allá 
de la existencia de una mayor vinculación con los actores del sistema y la revitalización de las instituciones científicas y tecnológicas, dentro del sector industrial no se registró un avance sustancial hacia mayores esfuerzos en las inversiones privadas en innovación que permitan identificar un proceso virtuoso en la generación de capacidades competitivas dinámicas. En este sentido, los datos muestran que sigue existiendo una fuerte concentración en torno a la adquisición de maquinaria y equipo, mayormente de origen extranjero, constituyendo un elemento crítico en la formación de la restricción externa al crecimiento sectorial.

\section{CONSIDERACIONES FINALES}

En primer lugar, y en torno al aspecto teórico conceptual, el artículo presenta una propuesta concreta para el abordaje de la problemática científica y tecnológica desde una perspectiva sistémica y contextualizada. En particular, la complementariedad teórica adquiere relevancia al relacionar el enfoque de SNI con la teoría de la regulación, en tanto permite identificar el conjunto de particularidades y especificidades nacionales para el estudio de la dimensión científica y tecnológica del desarrollo. En este sentido, se incorpora la esfera política de estudio desde la contextualización explícita en un modelo de desarrollo determinado por las formas de regulación y el régimen de acumulación en un momento histórico determinado. Es decir que el enfoque SNI se adhiere a estas categorías analíticas con foco en el carácter sistémico y científico- tecnológico, con un rol central del Estado como articulador mediante la formulación y ejecución de las políticas públicas.

En la identificación de los ciclos de divergencia y fortalecimiento entre la dinámica científica y tecnológica y los modelos de desarrollo para el caso argentino, fueron descriptas tres etapas. Una primera etapa de institucionalización, durante el período 1930-1976, abarcando las dos etapas del modelo ISI, donde se encuentra el origen de las principales instituciones, impulsadas por el Estado, el sector militar y en menor medida el sector productivo. Una segunda etapa de divergencia fue señalada para el período 1976-2002, contextualizada por la emergencia del neoliberalismo y de un nuevo paradigma tecnoeconómico, donde se adopta una visión ortodoxa de la producción y la movilidad del conocimiento y la tecnología, y por otro lado se produce la formalización SNI, expresando la divergencia entre políticas implícitas y explícitas en la materia. Por último, prologando los desafíos actuales, se identificó una etapa de fortalecimiento a partir de 2003, donde se advirtió una mayor consistencia en los lineamientos estratégicos hacia la consolidación del SNI. En este marco, se destacaron un conjunto de iniciativas y transformaciones que guiaron la orientación 
del desarrollo hacia la reducción de la divergencia entre el sistema de innovación y el modelo denominado neodesarrollista.

Respecto a los principales desafíos, se destaca la continuidad en la débil vinculación entre la sólida base de recursos científicos y tecnológicos y el sistema productivo en general. Se trata de una condición pendiente para la consolidación del SNI, asociada directamente al déficit de la dinámica del cambio tecnológico inducida desde la estructura productiva. Es decir, en un contexto donde la generación de ventajas competitivas dinámicas se encuentran relacionadas al desarrollo tecnológico y las innovaciones, las empresas no encuentran incentivos para convertirse en protagonistas del cambio tecnológico.

Si bien el crecimiento de la estructura productiva industrial constituyó un elemento central en el aumento de capacidades para el desarrollo tecnológico, desde el sector privado no se registraron mayores esfuerzos en sus inversiones en investigación y desarrollo, y en cuanto al desarrollo de actividades científicas y tecnológicas, se concentraron en la adquisición de bienes de producción, especialmente maquinaria y equipo.

El comportamiento de las principales empresas es clave en tanto constituyen actores centrales en su capacidad para dinamizar el cambio tecnológico. Sin embargo, en su desarrollo no necesitaron de un SNI, ni de la formación de recursos humanos calificados, sino, por el contrario, que basaron su obtención de beneficios sobre la disponibilidad de recursos naturales y en ventajas artificiales otorgadas a partir de subsidios, esquemas preferenciales, protecciones, concentración de mercados, libertades para fijar precios y un tipo de cambio competitivo.

A partir de allí se eleva el desafío de refundar el marco legal para regular la inversión extranjera y la actividad financiera que apunten a la consolidación del nuevo patrón de crecimiento, dado que aún se mantienen lineamientos propios de orden neoliberal que actúan en defensa del régimen de acumulación no basado en la innovación productiva. En este sentido, constituye también una oportunidad para fijar pautas de inversión en I+D y el compromiso de las firmas de acompañar el desarrollo de proveedores locales.

En suma, la principal reflexión es que si el régimen de acumulación no se motoriza en actividades asociadas a la innovación es el Estado, como formador y oferente de capacidades tecnológicas y como articulador en la generación de formas de regulación, quien debe promover su transformación mediante políticas públicas consistentes con un esquema de incentivos, orientándolo hacia formas dinámicas en la demanda y la 
generación de ventajas competitivas basadas en conocimiento.

Este desafío es clave no solo hacia la transformación estructural de largo plazo, sino que adquiere identidad inmediata bajo el riesgo de subutilización de los recursos científico y tecnológicos que se han generado en el período a partir del fuerte impulso estatal, puesto que puede derivar en las presiones políticas para la reasignación de los recursos y el consecuente debilitamiento de una de las bases fundamentales del desarrollo. 


\section{REFERENCIAS}

Albornoz, M. y Gordon, A. (2011). La Política Deficiencia y Tecnología en Argentina desde la Recuperación de la Democracia (1983-2009). En Albornoz Diéz-Rodríguez, M. y Sebastián, J. (Eds.), Trayectorias de las Politicas Cientificas y Universitarias de Argentina y España. Madrid: CSIC.

Araya, J. M. y Colombo, S. (2009). Economía, Estado y Sociedad en la Argentina a partir de las Reformas Estructurales. En Araya, J. M. (Comp.), Sociedad, Economía y Politica en la Argentina Contemporánea. Tandil: Graffikart.

Arocena, R. y Sutz, J. (2000). Looking at National Systems of Innovation from the South. Industry and Innovation, 7 (1), 55-75.

Basualdo, E. (2013). Estudios de Historia Económica Argentina: Desde Mediados del Siglo XX a la Actualidad. Buenos Aires: Siglo XXI Editores.

Bisang, R. (1995). Libremercado, Intervenciones Estatales e Instituciones de Ciencia y Técnica en la Argentina: Apuntes para una Discusión. Redes, Revista de Estudios Sociales de la Ciencia, 2 (3), 13-58.

Boyer, R. (2007). Crisis y Régimen de Crecimiento: Una Introducción a la Teoría de la Regulación. Buenos Aires: Miño y Dávila.

Bush, V. (1999). Ciencia, la Frontera sin Fin. Un Informe al Presidente, Julio de 1945. Redes. Revista de Estudios Sociales de la Ciencia, 6 (14), 89-137.

Chudnovsky, D. (1999). Políticas de Ciencia y Tecnología y el Sistema Nacional de Innovación en la Argentina. Revista de la CEPAL, 67, 153171.

Chudnovsky, D. y López, A. (1996). Política Tecnológica en la Argentina: ¿Hay Algo Más que Laissez-Faire? Redes, Revista de Estudios Sociales de la Ciencia, 3 (6), 33-75.

Consejo Nacional de Investigaciones Científicas y Técnicas (CONICET). (2006). Consejo Nacional de Investigaciones Cientificas y Técnicas. Buenos Aires: Editora y Empresa. 
Curia, E. (2011). El Modelo de Desarrollo en la Argentina. Los Riesgos de una Dinámica Pendular. Buenos Aires: Fondo de Cultura Económica.

De Angelis, I., Calvento, M., y Roark, M. (2013). ¿Hacia un Nuevo Modelo de Desarrollo? Desde la Teoría de la Regulación: Argentina 2003-2010. Problemas del Desarrollo, 44 (173), 31-56.

De Angelis, I. (2015). Dinámica del Cambio Tecnológico en el Modelo de Desarrollo Posconvertibilidad. Limites y Oportunidades para la Transformación de la Estructura Productiva Argentina: Un Análisis Contextualizado del Sector Maquinaria y Equipo. Tesis de Maestría (Ciencia, Tecnología y Sociedad), Universidad de Quilmes, Bernal, Argentina.

Dosi, G. (2003). Paradigmas Tecnológicos y Trayectorias Tecnológicas. La Dirección y los Determinantes del Cambio Tecnológico y la Transformación de la Economía. En Chesnais, F. y Neffa J. (Comp.), Ciencia, Tecnología y Crecimiento Económico. Buenos Aires: CEILPIETTE CONICET.

Edquist, C. (1997). Systems of Innovation: Technologies, Institutions and Organizations. Londres: Pinter Publishers

Freeman, C. (1995). The National System of Innovation in Historical Perspective. Cambridge Journal of Economics, 19 (1), 5-24

(2008). Systems of Innovation. Selected Essays in Evolutionary Economics Cheltenham. Northampton: Edward Elgar.

Freeman, C. y Pérez, C. (2003). Crisis Estructurales de Ajuste, Ciclos Económicos y Comportamiento de la Inversión. En Chesnais, F. y Neffa J. (Comp.), Ciencia, Tecnología y Crecimiento Económico. Buenos Aires: CEIL-PIETTE CONICET.

Gabinete Científico Tecnológico (GACTEC). (1997). Plan Nacional Plurianual de Ciencia y Tecnología 1998-2000. Buenos Aires: GACTEC.

Gordon, A. (2011). Políticas e Instrumentos en Ciencia, Tecnología e Innovación. Un Panorama sobre los Desarrollos Recientes en América Latina. Disponible en: http://www. politicascti.net/index.php?option $=$ com_docman\&task $=$ doc_ view\&gid=50\&Itemid=51\&lang=es [03-03-2015]. 
Gutti, P. (2008). Características del Proceso de Absorción Tecnológica de las empresas con Baja Inversión en I+D: Un Análisis de la Industria Manufacturera Argentina. Tesis de Maestría (Gestión de la Ciencia, la Tecnología y la Innovación), Universidad Nacional de General Sarmiento, Buenos Aires, Argentina.

Herrera, A. O. (1971). Ciencia y Política en América Latina. México: Siglo XXI.

Hervás, J. M., Rojas, R., Sempere, F. y Albors, J. (2012). Sistemas Nacionales de Innovación: Determinantes y Acciones de Política Industrial. Evidencia Empírica para la OCDE. Revista Economía Industrial, 383, 157-166.

Hurtado, D. (2014). Surgimiento, Alienación y Retorno. El Pensamiento Latinoamericano en Ciencia, Tecnología y Desarrollo. Voces en el Fénix, 2 (8), 21-27.

Instituto Nacional de Estadística y Censos (INDEC). (2008). Encuesta Nacional sobre Innovación y Conducta Tecnológica 2005. Buenos Aires: INDEC.

Kababe, Y. (2011). Aprendizaje por Interacción e Innovaciones Electrónicas en el Sector Agroindustrial Argentino. El Caso de la Empresa Sensor Automatización Agrícola. Tesis de Maestría (Gestión de la Ciencia, la Tecnología y la Innovación), Buenos Aires, Universidad Nacional de General Sarmiento.

Katz, J. (2000). Reformas Estructurales, Productividady Conducta Tecnológica en América Latina. Santiago: Fondo de Cultura Económica, CEPAL

Kline, S. y Rosenberg, N. (1986). An Overview of Innovation, in the Positive Sum Strategy: Harnessing Technology for Economic Growth. Washington: National Academy Press.

Lamarchand, G. (2010). Sistemas Nacionales de Ciencia, Tecnologia e Innovación en América Latina y el Caribe. Montevideo: UNESCO.

López, A. (2002). Industrialización Sustitutiva de importaciones y Sistema Nacional e Innovación: Un Análisis del Caso Argentino. Redes, Revista de Estudios Sociales de la Ciencia, 10 (19), 43-85. 
López, A. (2005). El Sistema Nacional de Innovación en la Argentina. Ponencia presentada en El Plan Fénix en Visperas del Segundo Centenario. Una Estrategia Nacional de Desarrollo con Equidad, 2-5 de agosto de 2005, Buenos Aires, Argentina.

Lugones, G. (2000). ¿Manual de Oslo o Manual Latinoamericano? Reflexiones a partir de la Encuesta Argentina sobre Conducta Tecnológica de las Firmas Industriales. Redes, Revista de Estudios Sociales de la Ciencia, 7 (16), 11-47.

. (2005). Sistema Nacional de Innovación y Desarrollo Económico en Argentina. Ponencia presentada en El Plan Fénix en Visperas del Segundo Centenario. Una Estrategia Nacional de Desarrollo con Equidad, 2-5 de agosto de 2005, Buenos Aires, Argentina.

Lundvall, B. (1992). National Systems of Innovation. Towards a Theory of Innovation and Interactive Learning. Londres: Pinter.

. (2009). Investigación en el Campo de los Sistemas de Innovación: Orígenes y Posible Futuro. Lundvall, B. (Ed.), Sistemas Nacionales de Innovación. Hacia una Teoría del Aprendizaje por Interacción. Buenos Aires: UNSAM.

Lundvall, B. y Johnson, B. (1994). Sistemas Nacionales de Innovación y Aprendizaje institucional. Comercio Exterior, 44 (8), 695-704.

Malerba, F. (2002). Sectoral Systems of Innovation and Production. Research Policy, 31 (2), 247-264.

Merton, R. (1977). La Sociología de la Ciencia. Madrid: Alianza Editorial.

Ministerio de Ciencia, Tecnología e Innovación Productiva de la Nación (MINCYT). (2015a). Encuesta Nacional de Dinámica de Empleo e Innovación. Sector Manufacturero 2010-2012. Buenos Aires: MINCYT.

(2015b). Encuesta I+D del Sector Empresario. Sistema Integrado de Indicadores CTI. Buenos Aires: MINCYT.

Navarro Arancegui, M. (2001). Los Sistemas Nacionales de Innovación: Una Revisión de la Literatura. Madrid: Instituto de Análisis Industrial y Financiero, Universidad Complutense Madrid. 
Neffa, J. C. (2006). Evolución Conceptual de la Teoría de la Regulación. De la Garza Toledo, E. (coord.). Teorias Sociales y Estudios del Trabajo: Nuevos Enfoques. Iztapalapa México: Anthropos. Barcelona: Coedición División de Ciencias Sociales y Humanidades. Universidad Autónoma Metropolitana.

Nochteff, H. (2002). ¿Existe una Política de Ciencia y Tecnología en la Argentina? Un Enfoque desde la Economía Política. Desarrollo Económico, 41 (164), 555-578.

Nun, J. (1995). Argentina: El Estado y las Actividades Científicas y Tecnológicas. Redes, Revista de Estudios Sociales de la Ciencia, 2 (3), 59-98.

Organización para la Cooperación y el Desarrollo Económico (OCDE). (1996). La Innovación Tecnológica: Definiciones y Elementos de Base. Redes, Revista de Estudios Sociales de la Ciencia, 3 (6), 131-175.

Pérez, C. (2001). Cambio Tecnológico y Oportunidades de Desarrollo como BLANCO MÓVIl. Revista de la CEPAL, 75,115-136.

. (2004). Revoluciones Tecnológicas y Capital Financiero: La Dinámica de las Grandes Burbujas Financieras y las Épocas de Bonanza. México: Siglo XXI.

Porta, F., y Bianco, C. (2004). Las Visiones sobre el Desarrollo Argentino. Consensos y Disensos. Documento de Trabajo 13. Buenos Aires: Centro Redes.

Pupato, G., Chudnovsky, D., y López, A. (2004). Sobreviviendo en la Convertibilidad. Innovación, Empresas Transnacionales y Productividad en la Industria Manufacturera. Desarrollo Económico, 44 (175), 365-395.

Rosegger, G. (1996). The Economics of Production and Innovation. An Industrial Perspective. Oxford: Butterworth-Heineman.

Schumpeter, J. (1943). Capitalism, Socialism and Democracy. Londres: McGraw-Hill.

Teece, D. (2003). Sacando Partido de la Innovación Tecnológica: Implicaciones para la Integración, Colaboración, Obtención de Licencias y Políticas Públicas. En Chesnais, F. (Ed.), Sistemas de 
Innovación y Política Tecnológica. Buenos Aires: Trabajo y Sociedad. Yoguel, G. (2000). Creación de Competencias en Ambientes Locales y Redes Productivas. Revista de la CEPAL, 71, 105-119.

Recibido: 19-05-2016

Aceptación de la versión final: 27-12-2017 